\title{
Fall Detection for Elderly People in Indoor Environment using Kinect Sensor
}

\author{
Disha Pathak ${ }^{1}$, V. K. Bhosale ${ }^{2}$ \\ ${ }^{1}$ Deogiri Institute of Engineering and Management Studies, Aurangabad, India \\ ${ }^{2}$ Department of Electronics and Telecommunication, Deogiri Institute of Engineering and Management Studies, Aurangabad, India
}

\begin{abstract}
Health problems of the elderly people are becoming more and more severe with the growth of aging population. The accidents like falling down are one of the major risks for the older adults living alone at home and need to be paid more attention. Through the analysis of the existing fall detection techniques, a more easy and quick algorithm about human body fall detection based on the human skeleton extraction using Kinect sensor is proposed in this paper. This algorithm consists of two parts, which are moving target depth of image acquisition, processing of depth image and identification of human skeleton. The realization of the detection algorithm is based on the tracked key joints acquired from the Kinect sensor. Two parameters are extracted, by comparing these values with the threshold, the system judges whether human falls down. Once a fall event is detected an alert message is sent to a predefined number using SIM900A GSM modem. Using new technologies such as the Kinect sensor with matlab could bring innovative ways to build smart systems that could use to observe the elderly people at homes and notify the caretakers in case of falling events Based on a real dataset of 50 people, experimental results indicate that the proposed method can realize human's fall detection with $94.65 \%$ accuracy in indoor environment.
\end{abstract}

Keywords: Kinect sensor, Fall detection, Depth image, Skeleton extraction

\section{Introduction}

Falls of elderly people is a major public problem. Many studies have indicated that falls in older adults are one of the most dangerous situations at home. Approximately 28-35\% of older adults fall one time or more per year. Moreover, falls are the foremost cause of accidental death in older adults aged 65 or more. When an elderly person living alone encounters a fall event, he/she may be lying on the floor for a long time without any assistance. This scenario mostly will lead to a severe negative outcome. Therefore, a fall accident detection system, which can automatically detect the fall accident and call for help, is very crucial for elderly people, especially for those living alone [1].Therefore, fall detection has attracted a large attention of researchers as well as industrial. In the last decade various material, equipment and fall detection methods have been proposed. Nowadays, fall detection approaches could be classified broadly as two main categories: non-vision-based method and vision-based method. Among these methods, vision based method has a prominent advantage since it does not require the older adults to wear any specific equipment.

Recently, Kinect sensor has been released. The utilization of this sensor for health care industry and fall detection in particular has three main advantages. Firstly, this sensor provide useful details (color, depth and skeleton) in contrast to conventional 2D cameras and therefore gives a better representation of fall events. Secondly, this device allows developing a solution for protecting privacy of the elderly people - major issue in health care application by using only depth and skeleton information [2]. Also the Kinect sensor is independent of lighting conditions, since it is equipped with an active light source. As the Kinect utilizes infrared light it is possible to extract depth images in a complete dark room too.
In this paper, we present a real time fall detection system based on the Kinect device. The originalities of this system are two-fold. Firstly key joints of the human body is tracked using a single depth camera i.e Microsoft Kinect . Secondly the head and the hip joint is extracted and compared with a threshold value. Based on the comparison, system makes a decision whether human fall down or not. Thereafter an alert message indicating the person fallen state is sent to a predefined number. The approach of detecting falls presented in this paper is unobtrusive, which does not have any environmental interaction with the target people.

\section{Related Work}

The fall detection system uses varied techniques and technologies. These techniques and technologies are categorized as vision and non-vision based groups. These techniques have their own benefits as well as disadvantages. Though non vision-based methods appear to be best suited for large application in the fall detection field, some problems do exist; they are easily affected by noise in the environment (acoustic sensors and floor vibration sensors) or either inconvenient (elderly people have to wear acceleration sensors). To overcome these issues, vision-based fall detection techniques are adopted. These systems have a foremost benefit over non vision system. In these methods it is not mandatory to wear any devices. Here one or more cameras are used to track people. Cameras can be used to detect multiple events simultaneously with very less intrusion. However Personal privacy is a concerning issue for older adults in vision-based fall detection systems. In most computer vision-based fall detection systems, only the alarm signal will be sent to the caregivers or family members when a fall event is detected; furthermore, the original video recordings of the older adult's normal activities will neither be stored, nor transmitted [3-5]. Vision based methods can be 


\section{International Journal of Science and Research (IJSR) \\ ISSN (Online): 2319-7064 \\ Index Copernicus Value (2015): 78.96 Impact Factor (2015): 6.391}

performed either of the three ways:

- Methods using a single RGB camera

- 3D- based methods using multiple cameras

- 3D- based methods that use depth cameras

However, the existing video-based devices for detecting fall events cannot work properly in nightlight or low light conditions. In addition, in most of such solutions the privacy of the person is not preserved adequately. Recently, Kinect sensor has been proposed to achieve fall detection. The Kinect is a revolutionary motion-sensing technology that allows tracking a person in real-time without having to carry sensors.

The earliest depth camera used for fall detection is the Time Of-Flight 3D camera [6]. Since the price of a Time-Of Flight $3 \mathrm{D}$ camera is expensive, very few researchers adopted it for fall detection. However this situation has changed since the advent of the affordable depth sensing technology, like Microsoft Kinect. Over the last few years, there are a number of works that have been proposed for detecting fall events using Kinect sensor. These works can be categorized according to whether they focus on direct detection of the critical phase/impact shock or postfall phase.

Some recent reviews as given below explains various methods of fall detection using Kinect sensor. The Kinectbased fall detection work can be classified according to the information source. The work corresponding to the first category makes use of the color and depth information while the second category utilizes skeleton information to detect the fall.

This work demonstrates the advancement of the Kinect based fall detection systems.

Table 1: Comparison of Few Different Approaches

\begin{tabular}{|c|c|c|c|c|}
\hline Sr. no & Title & Detection technique & Type of sensor & Remarks \\
\hline 1. & $\begin{array}{l}\text { An analysis on human fall } \\
\text { detection using skeleton from } \\
\text { Microsoft Kinect [2] }\end{array}$ & $\begin{array}{l}\text { Support Vector Machine technique is used } \\
\text { to distinguish fall with other activities }\end{array}$ & Microsoft Kinect & $\begin{array}{c}\text { Three features (distance, angle, } \\
\text { velocity) are defined and } \\
\text { computed for few important } \\
\text { joints }\end{array}$ \\
\hline 2. & \begin{tabular}{|c|} 
Fall Detection in Homes of Older \\
Adults Using the Microsoft Kinect \\
{$[7]$}
\end{tabular} & $\begin{array}{c}1^{\text {st }} \text { Stage - Vertical state characterization } \\
2^{\text {nd }} \text { Stage - On ground event features, } \\
\text { ensemble for fall confidence } \\
\end{array}$ & Microsoft Kinect & $\begin{array}{c}\text { Decreased resolution of faller } \\
\text { makes foreground segmentation } \\
\text { difficult. } \\
\end{array}$ \\
\hline 3. & $\begin{array}{l}\text { A Vision based Fall Detection } \\
\text { System for Elderly People [8] }\end{array}$ & $\begin{array}{c}\text { Canny Algorithm, Contour Approximation } \\
\text { Method } \\
\end{array}$ & Microsoft Kinect & $\begin{array}{c}\text { The Raspberry pi and Open CV } \\
1.0 \text { are used } \\
\end{array}$ \\
\hline 4. & $\begin{array}{c}\text { An active vision system for fall } \\
\text { detection and posture recognition } \\
\text { in elderly healthcare [9] }\end{array}$ & People's silhouette and their centre-of-mass & TOF camera & $\begin{array}{l}\text { Cost of TOF camera. } \\
\text { Orientation of spine from the } \\
\text { extracted skeleton is needed. }\end{array}$ \\
\hline 5. & \begin{tabular}{|c|} 
A Real-Time Fall Detection System \\
in Elderly Care Using Mobile \\
Robot and Kinect Sensor [10]
\end{tabular} & $\begin{array}{l}\text { Kinect sensor is used with mobile robot } \\
\text { system to follow a person and detect fall } \\
\text { events. }\end{array}$ & Microsoft Kinect & Need for the mobile robot system \\
\hline 6. & $\begin{array}{c}\text { Fuzzy inference-based fall } \\
\text { detection using kinect and body- } \\
\text { worn accelerometer [11] }\end{array}$ & $\begin{array}{c}\text { A fuzzy system consisting of two input } \\
\text { Mamdani engines and a triggering alert } \\
\text { Sugeno engines is used to detect fall events }\end{array}$ & \begin{tabular}{|c|}
$\begin{array}{c}\text { Kinect and Body- } \\
\text { worn } \\
\text { Accelerometer }\end{array}$ \\
\end{tabular} & $\begin{array}{l}\text { Accelerometer may Provide } \\
\text { discomforts }\end{array}$ \\
\hline
\end{tabular}

\section{System Development}

In the proposed method MATLAB R2013 tool is used for processing of data which is acquired from the kinect senor. With matlab, the Kinect studio version 1.8.0 is used to access the depth data and for skeleton extraction. Fig.1 shows the proposed fall detection system.

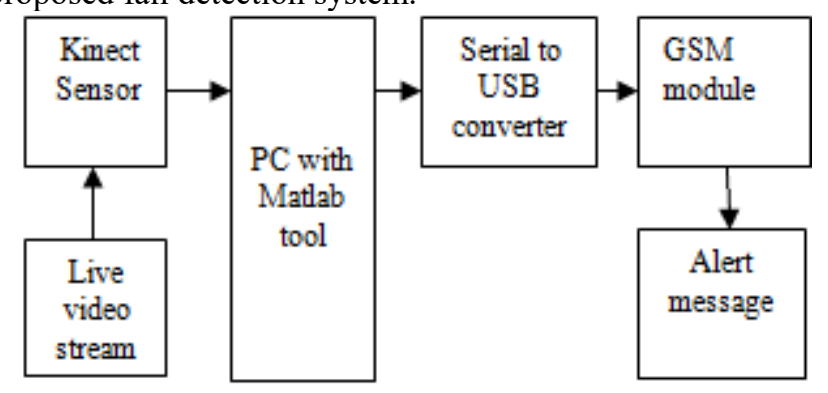

Figure 1: Proposed Fall Detection system

\subsection{Kinect Sensor}

Microsoft Kinect is essentially a set of sensors that was formerly developed as an input tool for the Xbox 360 and Xbox One gaming console .It comprise of the infrared projector with Quarter Video Graphics Array (QVGA) (640x480) IR camera which is utilized for getting precise depth maps, a RGB color camera VGA (640x480), a fourmicrophone array and a motorized tilt. These are combined altogether to form various numbers of image processing applications. Image streams at 30 frames per second (fps) are produced in both cameras.

With the help of depth sensors, the Kinect can recognize up to six users standing from 0.8 to 4.0 meters (i.e 2.6 to 13.1 feet). Furthermore two of the detected skeleton can also be tracked in aspect with 20 joint positions. Each skeleton joint is calculated in a three dimensional $(\mathrm{X}, \mathrm{Y}, \mathrm{Z})$ plane. $\mathrm{X}$ - axis 


\section{International Journal of Science and Research (IJSR)}

ISSN (Online): 2319-7064

Index Copernicus Value (2015): 78.96 | Impact Factor (2015): 6.391

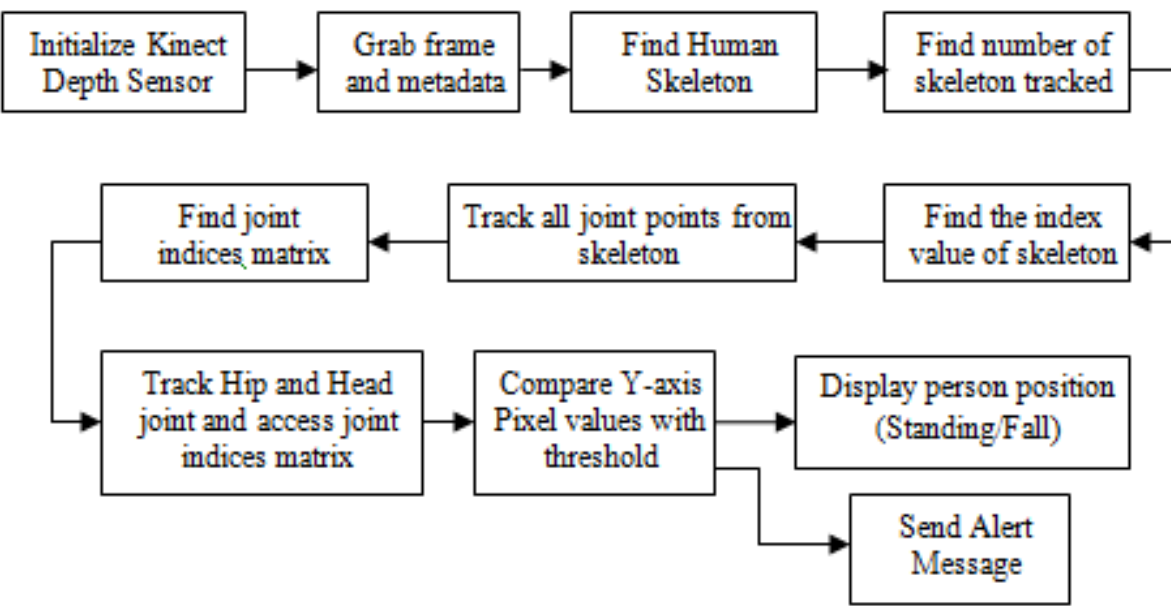

Figure 2: Data Flow of Proposed Fall Detection system

will vary when the joints move from the right side to the left side or vice versa, while the $\mathrm{Y}$ axis will vary when the joints move either in the upwards or downwards direction. Similarly, $\mathrm{Z}$ axis will vary when the joint is moved back and forth in relation to the Kinect sensor.

\subsection{SIM900A GSM modem}

SIM900A GSM has 68 Surface Mount Technology (SMT) pads and provides interfaces between the module and all user's hardware. This is an ultra compact and reliable wireless module. SIM900A GSM modem is regulated by the AT (ATtention) command and is set to the SMS text mode to send an SMS message. Thus, SMS would be sent to a predefined person when the fall is identified.

Few of SIM900A features are as follows:

- Dual band GSM/GPRS 900/1800MHz.

- Configurable baud rate.

- Low power consumption: $1.5 \mathrm{~mA}$ (sleep mode)

- Built in network status LED.

\subsection{Proposed Algorithm}

The proposed algorithm is based on depth map and makes use of a Microsoft Kinect device. Here system will detect the skeletal data to recognize older adults and track their actions. In this work it is assumed that the elderly person is completely perceptible and do not have any occlusions. The Kinect sensor is steady and the movement of the subject along the sensor is restrained to a range. An overview of the proposed fall detection method is as shown.

The logical steps on which the proposed system is built are as follows:

\section{Results}

The proposed method is implemented in MATLAB R2013 using the Open Kinect libraries. Some of the results of fall detection are shown in figure. Evaluation of the proposed fall detection system
Table 2: Characteristics of 50 participators in our experiment

\begin{tabular}{|c|c|}
\hline No. of People & 50 \\
\hline Male/Female & $25 / 25$ \\
\hline Age & $21-60$ \\
\hline Weight & $53-94(\mathrm{Kg})$ \\
\hline Height & $5.1-6.2 \mathrm{ft}$ \\
\hline
\end{tabular}

The proposed algorithm is being tested at different time and lighting condition. The Table 3 show the accuracies of the proposed fall detection algorithm.

Table 3: Percentage Success of different positions

\begin{tabular}{|c|c|c|c|c|}
\hline Category & $\begin{array}{c}\text { No. of } \\
\text { activities }\end{array}$ & $\begin{array}{c}\text { Fall acceptance } \\
\text { rate }\end{array}$ & $\begin{array}{c}\text { Fall rejection } \\
\text { rate }\end{array}$ & $\begin{array}{c}\% \\
\text { Success }\end{array}$ \\
\hline Falls & 150 & 143 & 7 & $95.33 \%$ \\
\hline Walking around & 80 & 0 & 80 & $100 \%$ \\
\hline Lying on sofa & 60 & 6 & 54 & $90 \%$ \\
\hline Sitting on chair & 60 & 4 & 56 & $93.33 \%$ \\
\hline
\end{tabular}

During the evaluation the distance of the Kinect sensor was changed from $0.8 \mathrm{~m}$ to $4.5 \mathrm{~m}$, which is technically its sensing range. The results obtained are summarized in Figure 4. The figure shows, when the fixed distance was between $1.5 \mathrm{~m}$ to $3 \mathrm{~m}$, the fall detection rate was the highest. Figure below shows the fall detection rate. Here the y axis represents the fall detection rate $(\%)$, whereas the $\mathrm{x}$-axis denotes the distance between the sensor and subject in meters.

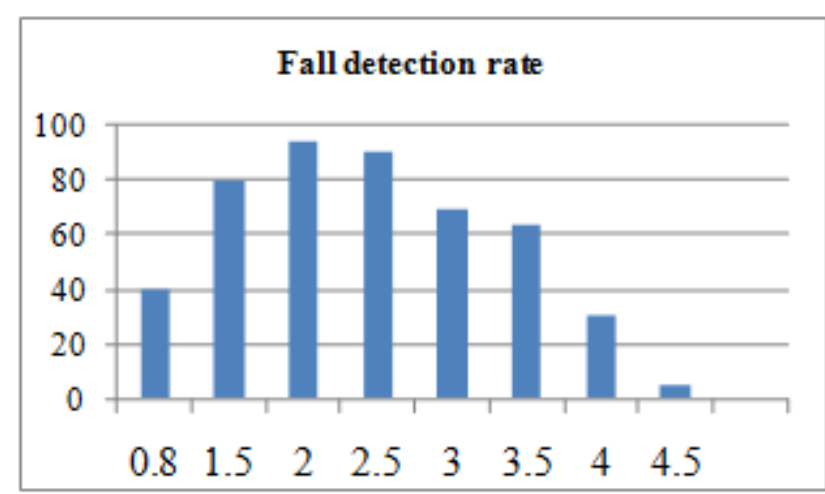

Figure 3: Fall detection rate (Different distance) 


\section{International Journal of Science and Research (IJSR) \\ ISSN (Online): 2319-7064}

Index Copernicus Value (2015): 78.96 | Impact Factor (2015): 6.391

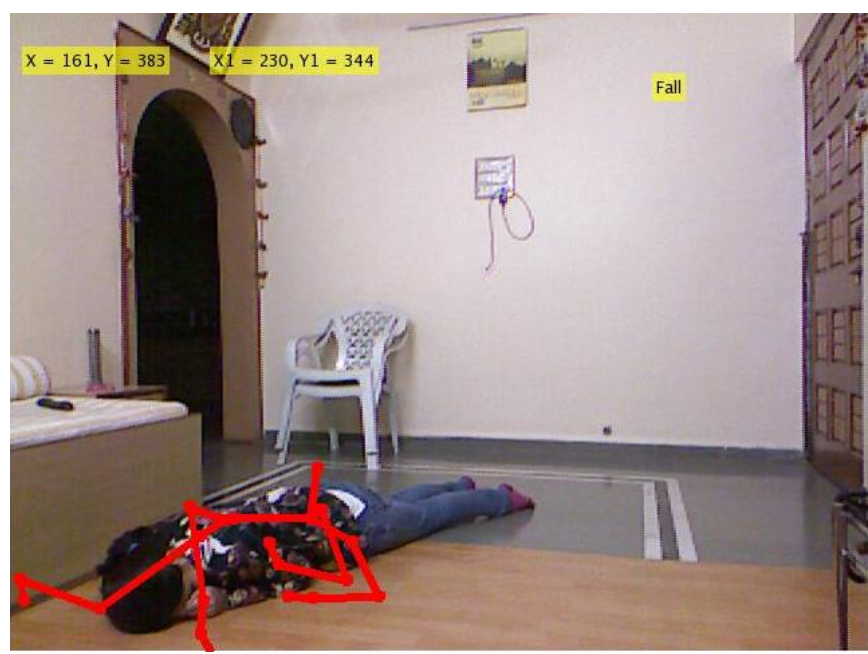

Figure 4: Fallen Person in color image

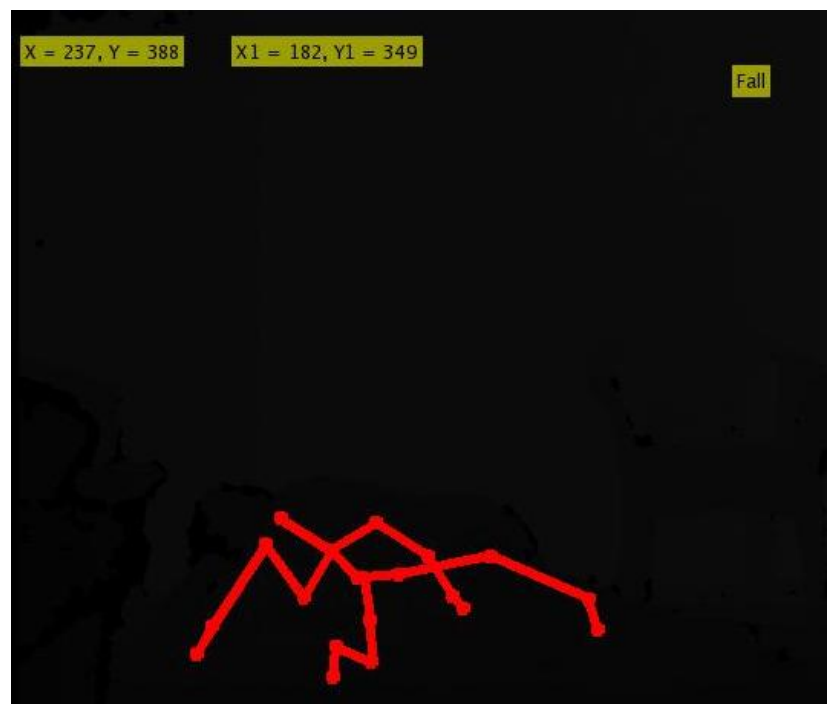

Figure 5: Fallen Person in depth image

Above Figure (4-5) shows the color and depth images of captured data with Skeleton tracking. The live images are captured and featured by the fall detection algorithm. The images are taken for testing and the following result is displayed.

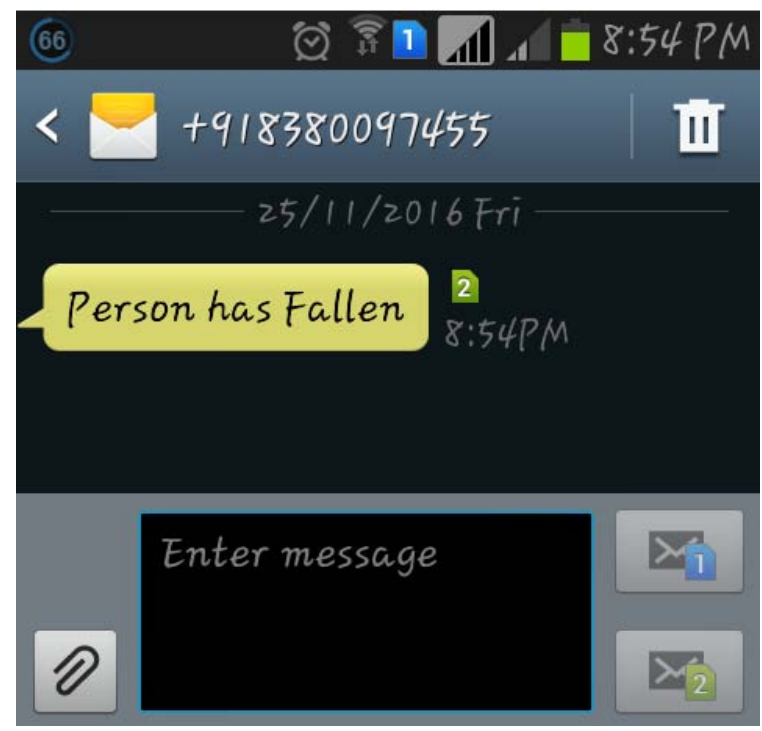

Figure 6: Alert SMS message

\section{Conclusion}

In this paper, we proposed a fall detection system for elderly people based on the skeleton extraction using Kinect sensor. The proposed fall detection approach uses the infrared based depth camera, so the system can operate even in the dark condition. Kinect sensor is mature and cheap. So the system can easily be applied in smart home environment. The system can also be used with elderly people using crutches, canes, and walkers and works reliably. The proposed system supports the independent living of elderly people and can be implemented in particular scenarios as hospitals, recreation centers for the elderly and domestic houses. Based on depth image sequence, by extracting and tracking the joints of the human body as well as investigating the joints' behaviour after fall, the proposed approach can detect and confirm the human fall accurately. The experimental results demonstrate that our proposed method can accomplish near $94.65 \%$ frame accuracy.

Future work includes advancing the minor movement tracking of elderly people and updating the status to the caretakers using cloud services. Also using GPS the latitude and longitude of the fallen person can be extracted, this information then can be directly sent to the nearby hospitals. In this work, the kinect v1 sensor is used. In future, the kinect v2 sensor can be used to enhance the resolution of the depth image. The use of kinect for fall detection was quite satisfactory, but future work requires an additional upgrading in the fall detection methodology.

\section{References}

[1] Bian, Zhen-Peng, et al. "Fall detection based on body part tracking using a depth camera." IEEE journal of biomedical and health informatics 19.2 (2015): 430439.J. Clerk Maxwell, A Treatise on Electricity and Magnetism, 3rd ed., vol. 2. Oxford: Clarendon, 1892, pp.68-73.

[2] Le, Thi-Lan, and Jeremy Morel. "An analysis on human fall detection using skeleton from Microsoft Kinect." Communications and Electronics (ICCE), 2014 IEEE Fifth International Conference on. IEEE, 2014.

[3] Mubashir, Muhammad, Ling Shao, and Luke Seed. "A survey on fall detection: Principles and approaches." Neurocomputing 100 (2013): 144-152.

[4] $\mathrm{Yu}, \mathrm{Miao}$, et al. "A posture recognition-based fall detection system for monitoring an elderly person in a smart home environment." IEEE transactions on information technology in biomedicine 16.6 (2012): 1274-1286.

[5] Delahoz, Yueng Santiago, and Miguel Angel Labrador. "Survey on fall detection and fall prevention using wearable and external sensors." Sensors14.10 (2014): 19806-19842.

[6] Zhang, Zhong, Christopher Conly, and Vassilis Athitsos. "A survey on vision-based fall detection." Proceedings of the 8th ACM International Conference on PErvasive Technologies Related to Assistive Environments. ACM, 2015. 
[7] Stone, Erik E., and Marjorie Skubic. "Fall detection in homes of older adults using the Microsoft Kinect." IEEE journal of biomedical and health informatics 19.1 (2015): 290-301.

[8] Madhubala, J. Sree, and A. Umamakeswari. "A vision based fall detection system for elderly people." Indian Journal of Science and Technology 8 (2015): 167.

[9] Diraco, Giovanni, Alessandro Leone, and Pietro Siciliano. "An active vision system for fall detection and posture recognition in elderly healthcare." 2010 Design, Automation \& Test in Europe Conference \& Exhibition (DATE 2010). IEEE, 2010.

[10] Mundher, Zaid A., and Jiaofei Zhong. "A real-time fall detection system in elderly care using mobile robot and kinect sensor." International Journal of Materials, Mechanics and Manufacturing 2.2 (2014): 133-138.

[11] Kwolek, Bogdan, and Michal Kepski. "Fuzzy inferencebased fall detection using kinect and body-worn accelerometer." Applied Soft Computing 40 (2016): 305-318. 